\title{
On values of the Mahler measure in a quadratic field (solution of a problem of Dixon and Dubickas)
}

\author{
by
}

\author{
A. Schinzel (Warszawa)
}

To Robert Tijdeman on the occasion of his 60th birthday

For an algebraic number $\alpha$, let $M(\alpha)$ be the Mahler measure of $\alpha$ and let $\mathcal{M}=\{M(\alpha) \mid \alpha \in \overline{\mathbb{Q}}\}$. No method is known to decide whether a given algebraic integer $\beta$ is in $\mathcal{M}$. Partial results have been obtained by Adler and Marcus [1], Boyd [2]-[4], Dubickas [6]-[8] and Dixon and Dubickas [5], but the problem has not been solved even for $\beta$ of degree two. The following theorem, similar to, but not identical with Theorem 9 of [5], is an easy consequence of [7].

TheOREM 1. A primitive real quadratic integer $\beta$ is in $\mathcal{M}$ if and only if there exists a rational integer a such that $\beta>a>\left|\beta^{\prime}\right|$ and $a \mid \beta \beta^{\prime}$, where $\beta^{\prime}$ is the conjugate of $\beta$. If the condition is satisfied, then $\beta=M(\beta / a)$ and $a=N(a, \beta)$, where $N$ denotes the absolute norm.

There remain to be considered quadratic integers that are not primitive. The following theorem deals with the simplest class of such numbers.

Theorem 2. Let $K$ be a quadratic field with discriminant $\Delta>0, \beta, \beta^{\prime}$ be conjugate primitive integers of $K$ and $p$ a prime. If

$$
p \beta \in \mathcal{M},
$$

then either there exists an integer $r$ such that

$$
p \beta>r>p\left|\beta^{\prime}\right| \text { and } r \mid \beta \beta^{\prime}, \quad p \nmid r
$$

or

$$
\beta \in \mathcal{M} \text { and } p \text { splits in } K \text {. }
$$

2000 Mathematics Subject Classification: Primary 11R04. 
Conversely, (2) implies (1), while (3) implies (1) provided either

$$
\beta>\max \left\{-4 \beta^{\prime},\left(\frac{1+\sqrt{\Delta}}{4}\right)^{2}\right\}
$$

or

$$
p>\sqrt{\Delta}
$$

REMARK 1. (2) implies $\beta>p \beta\left|\beta^{\prime}\right| / r \geq p$.

Theorem 2 answers two questions raised in [5].

Corollary 1. For all primes $p$ we have $p \frac{3+\sqrt{5}}{2} \in \mathcal{M}$ if and only if either $p=2$, or $p=5$, or $p \equiv \pm 1(\bmod 5)$.

Corollary 2. For every real quadratic field $K$ there is an irreducible polynomial $f \in \mathbb{Z}[x]$, basal in the sense of $[5]$, such that $M(f) \in K$, but the zeros of $f$ do not lie in $K$.

COROLlary 3. In every real quadratic field $K$ there are only finitely many integers $p \beta$, where $p$ is prime, while $\beta$ is primitive and totally positive, for which the condition $p \beta \in \mathcal{M}$ is not equivalent to the alternative of (2) and (3).

Proof of Theorem 1. Necessity. Let $\beta=M(\alpha)$, let $f$ be the minimal polynomial of $\alpha$ over $\mathbb{Z}, a>0$ its leading coefficient, $D$ its degree, and $\alpha_{1}, \ldots, \alpha_{D}$ all its zeros. By Lemma 2 of [7] applied with $d=2$,

$$
\beta \beta^{\prime}=a^{2} \prod_{i=1}^{D} \alpha_{i}=(-1)^{D} a f(0) .
$$

Moreover, by formula (3) of [7], $D=2 s$, where $s$ is the number of $i \leq D$ with $\left|\alpha_{i}\right|>1$. Without loss of generality we may assume that $\left|\alpha_{i}\right|>1$ precisely for $i \leq s$. For some $\eta \in\{1,-1\}$ we have

hence, by (6),

$$
\prod_{i=1}^{s} \alpha_{i}=\eta \beta / a
$$

which gives

$$
\prod_{i=s+1}^{D} \alpha_{i}=\eta \beta^{\prime} / a
$$

$$
\beta>a>\left|\beta^{\prime}\right|
$$

Also, by (6),

$$
a \mid \beta \beta^{\prime}
$$

Sufficiency. Assume the existence of an integer a satisfying (9) and (10) and consider the polynomial 


$$
g(x)=a x^{2}-\left(\beta+\beta^{\prime}\right) x+\beta \beta^{\prime} / a .
$$

If $g$ is not primitive, there exists a prime $p$ such that $p|a, p| \beta+\beta^{\prime}$ and $p \mid \beta \beta^{\prime} / a$. However, then $p^{2} \mid \beta \beta^{\prime}$ and $\beta / p$ is a zero of the polynomial $x^{2}-$ $\frac{\beta+\beta^{\prime}}{p} x+\frac{\beta \beta^{\prime}}{p^{2}} \in \mathbb{Z}[x]$, contrary to the assumption that $\beta$ is primitive. Therefore, $g$ is the minimal polynomial of $\beta / a$ over $\mathbb{Z}$ and $\beta=M(\beta / a)$. Also, (a) $\left|\left(a^{2}, a \beta, a \beta^{\prime}, \beta \beta^{\prime}\right)\right|\left(a^{2}, a\left(\beta+\beta^{\prime}\right), \beta \beta^{\prime}\right)=(a)$, hence

$$
(a)=\left(a^{2}, a \beta, a \beta^{\prime}, \beta \beta^{\prime}\right)=(a, \beta)\left(a, \beta^{\prime}\right) .
$$

The proof of Theorem 2 is based on three lemmas.

Lemma 1. If an integer $\beta$ of $K$ is the Mahler measure of an algebraic number whose minimal polynomial over $\mathbb{Z}$ has leading coefficient $a$, then a is the norm of an ideal of $K$.

Proof. In the notation of the proof of Theorem 1 (necessity part) we have (7) and (8). Since $\eta \beta^{\prime} / a$ is the only conjugate of $\eta \beta / a$, every automorphism of the splitting field of $f$ that sends an $\alpha_{i}(i \leq s)$ to an $\alpha_{j}(j>s)$ sends the set $\left\{\alpha_{1}, \ldots, \alpha_{s}\right\}$ onto $\left\{\alpha_{s+1}, \ldots, \alpha_{D}\right\}$ (compare the proof of Lemma 2 in [7]). Hence $\left\{\alpha_{1}, \ldots, \alpha_{s}\right\}$ and $\left\{\alpha_{s+1}, \ldots, \alpha_{D}\right\}$ are blocks of imprimitivity of the Galois group of $f$ and the coefficients of the polynomials

$$
P(x)=\prod_{i=1}^{s}\left(x-\alpha_{i}\right), \quad P^{\prime}(x)=\prod_{i=s+1}^{D}\left(x-\alpha_{i}\right)
$$

belong to a quadratic field, which clearly is $K$. Let the contents of $P$ and $P^{\prime}$ be $\mathfrak{a}^{-1}$ and $\mathfrak{a}^{\prime-1}$, where $\mathfrak{a}$ and $\mathfrak{a}^{\prime}$ are conjugate ideals of $K$. Since $f$ is primitive, we have

$$
(1)=\operatorname{cont} f=\operatorname{cont}\left(a P P^{\prime}\right)=(a) / \mathfrak{a} \mathfrak{a}^{\prime}
$$

and, since $a>0, a=N \mathfrak{a}$.

Lemma 2. If the dash denotes conjugation in $K, \delta, \varepsilon$ are elements of $K$ such that

$$
\begin{gathered}
\delta>1>\delta^{\prime}>-1 / 2, \\
(1, \delta) \mid \varepsilon, \quad \varepsilon \neq \varepsilon^{\prime}, \\
\left|\varepsilon-\varepsilon^{\prime}\right|+1<4 \sqrt{\delta}
\end{gathered}
$$

while $\mathfrak{p}$ is an ideal of $K$, then there exists $\gamma \in K$ such that

$$
\begin{gathered}
(1, \gamma, \delta)=\frac{(1, \delta)}{\mathfrak{p}}, \\
|\gamma|<2 \sqrt{\delta}, \quad\left|\gamma^{\prime}\right|<1+\delta^{\prime} .
\end{gathered}
$$


Proof. Take an integer $\alpha$ of $K$ divisible by $\mathfrak{p}(1, \delta)^{-1}$. Applying Theorem 74 of [9] with

$$
\mathfrak{a}=\frac{(\alpha)(1, \delta)}{\mathfrak{p}}, \quad \mathfrak{b}=\frac{\mathfrak{p}}{(1, \delta)}
$$

we find an integer $\omega$ of $K$ such that $(\alpha, \omega)=\mathfrak{a}$, hence

$$
\left(1, \frac{\omega}{\alpha}\right)=\frac{(1, \delta)}{\mathfrak{p}} \text {. }
$$

Taking

$$
b=\left\lfloor\left(\frac{\omega}{\alpha}-\frac{\omega^{\prime}}{\alpha^{\prime}}\right) /\left(\varepsilon-\varepsilon^{\prime}\right)+\frac{1}{2}\right\rfloor, \quad a=\left\lfloor\frac{\omega^{\prime}}{\alpha^{\prime}}-b \varepsilon^{\prime}+\frac{1}{2}\right\rfloor
$$

we find

$$
\left|\frac{\omega}{\alpha}-\frac{\omega^{\prime}}{\alpha^{\prime}}-b\left(\varepsilon-\varepsilon^{\prime}\right)\right| \leq \frac{\left|\varepsilon-\varepsilon^{\prime}\right|}{2}, \quad\left|\frac{\omega^{\prime}}{\alpha^{\prime}}-a-b \varepsilon^{\prime}\right| \leq \frac{1}{2}<1+\delta^{\prime},
$$

hence on addition, by (13),

$$
\left|\frac{\omega}{\alpha}-a-b \varepsilon\right| \leq \frac{\left|\varepsilon-\varepsilon^{\prime}\right|}{2}+\frac{1}{2}<2 \sqrt{\delta}
$$

and for $\gamma=\omega / \alpha-a-b \varepsilon$, (14) follows from (16), while (15) from (17) and (18).

Lemma 3. If , in the notation of Lemma $2, \mathfrak{p}$ is a prime ideal dividing a rational prime $p$, then the conclusion of the lemma holds, provided

$$
p>\frac{N(1, \delta) \sqrt{\Delta}}{\min \left\{N(1, \delta), 2 \sqrt{\delta}\left(1+\delta^{\prime}\right)\right\}} .
$$

Proof. Let the ideal $(1, \delta)$ considered as a module over $\mathbb{Z}$ have the basis $[\eta, \zeta]$. The system of inequalities

$|c|<p, \quad\left|c \frac{\omega}{\alpha}-a \eta-b \zeta\right|<2 \sqrt{\delta}, \quad\left|c \frac{\omega^{\prime}}{\alpha^{\prime}}-a \eta^{\prime}-b \zeta^{\prime}\right|<\min \left\{\frac{N(1, \delta)}{2 \sqrt{\delta}}, 1+\delta^{\prime}\right\}$ has a non-zero integer solution by Minkowski's theorem (Theorem 94 of [9]), since by Theorem 76 of [9], which applies also to fractional ideals (see $\S 31$, formula (47))

$$
\left|\eta \zeta^{\prime}-\eta^{\prime} \zeta\right|=N(1, \delta) \sqrt{\Delta}<\min \left\{N(1, \delta), 2 \sqrt{\delta}\left(1+\delta^{\prime}\right)\right\} p .
$$

If in this solution we had $c=0$ it would follow that $a \eta+b \zeta \neq 0$ and

$$
N(1, \delta) \leq|N(a \eta+b \zeta)|<2 \sqrt{\delta} \frac{N(1, \delta)}{2 \sqrt{\delta}}=N(1, \delta),
$$

a contradiction. Therefore $c \neq 0, c \not \equiv 0(\bmod \mathfrak{p})$ and $\gamma=c \frac{\omega}{\alpha}-a \eta-b \zeta$ has the required properties. 
Proof of Theorem 2. Assume first that (1) holds and let $f$ be the minimal polynomial of $\alpha$ over $\mathbb{Z}, a>0$ its leading coefficient, and $D$ its degree. By (6) and (7) with $\beta$ replaced by $p \beta$, we have

$$
\begin{gathered}
p^{2} \beta \beta^{\prime}=(-1)^{D} a f(0), \\
p \beta>\max \{a,|f(0)|\} \geq \min \{a,|f(0)|\}>p\left|\beta^{\prime}\right| .
\end{gathered}
$$

Let $p^{\mu}\left\|a, p^{\nu}\right\| \beta \beta^{\prime}$. If $\mu=0$ or $\mu=\nu+2$, then (2) follows with $r=a$ or $r=|f(0)|$, respectively. Therefore, assume

$$
1 \leq \mu \leq \nu+1 .
$$

Let $a=p^{\mu} b$. By (20) and (22),

$$
p^{\mu-1} b \mid \beta \beta^{\prime}
$$

while by (21),

$$
\beta>p^{\mu-1} b>\left|\beta^{\prime}\right| .
$$

By Theorem 1 we have $\beta \in \mathcal{M}$. If $\nu>0$, then $p \mid \beta \beta^{\prime}$ and since $\beta$ is primitive, $p$ splits in $K$. If $\nu=0$ we have, by (22), $\mu=1$ and since, by Lemma $1, a$ is the norm of an ideal of $K, p$ splits in $K$. This proves (3).

In the opposite direction, (2) implies $p \beta=M(p \beta / r) \in \mathcal{M}$. Indeed, the minimal polynomial of $p \beta / r$ is $r x^{2}-p\left(\beta+\beta^{\prime}\right) x+\beta \beta^{\prime} / r$, where $\left(r, \beta+\beta^{\prime}\right.$, $\left.\beta \beta^{\prime} / r\right)=1$, since $\beta$ is primitive (see the proof of Theorem 1). Assume now that (3) holds. By Theorem 1 we have $\beta=M(\beta / b)$, where

$$
b \in \mathbb{N}, \quad \beta>b>\left|\beta^{\prime}\right|, \quad b=N(b, \beta) .
$$

Replacing $b$ by $\beta\left|\beta^{\prime}\right| / b$ if necessary, we may assume

$$
b \geq \sqrt{\beta\left|\beta^{\prime}\right|}
$$

First, assume (4). Since $\beta$ is primitive all prime ideal factors of $(b, \beta)$ are of degree one and no two of them are conjugate. Hence there exists $c \in \mathbb{Z}$ such that

$$
\omega:=\frac{\Delta+\sqrt{\Delta}}{2} \equiv-c(\bmod (b, \beta)) .
$$

We put $\delta=\beta / b, \varepsilon=(c+\omega) / b$. In order to apply Lemma 2 we have to check the assumptions. Now, (11) follows from (23), (24) and $\beta>-4 \beta^{\prime}$, (12) follows from (25), and (13) is equivalent to the inequality

$$
\sqrt{\Delta} / \sqrt{b}+\sqrt{b}<4 \sqrt{\beta} .
$$

The left-hand side considered as a function of $b$ on the interval $[1, \beta]$ takes its maximum at an end of the interval. We have $\sqrt{\Delta}+1<4 \sqrt{\beta}$ by (4) and $\sqrt{\Delta} / \sqrt{\beta}+\sqrt{\beta}<4 \sqrt{\beta}$ since $\beta \geq(1+\sqrt{\Delta}) / 2$. 
The assumptions of Lemma 2 being satisfied there exists $\gamma \in K$ such that

$$
(1, \gamma, \delta)=\frac{(b, \beta)}{(b) \mathfrak{p}}=\frac{1}{\left(b, \beta^{\prime}\right) \mathfrak{p}}, \quad|\gamma|<2 \sqrt{\delta}, \quad\left|\gamma^{\prime}\right|<1+\delta^{\prime} .
$$

Let us consider the polynomial

$$
P(x)=x^{2}+\gamma x+\delta .
$$

The discriminant of $P, \gamma^{2}-4 \delta$, is negative, hence $P$ is irreducible over the real field $K$, moreover its zeros are equal to $\sqrt{\delta}>1$ in absolute value. On the other hand, the zeros of the polynomial

$$
P^{\prime}(x)=x^{2}+\gamma^{\prime} x+\delta^{\prime}
$$

are less than 1 in absolute value. This is clear if $\gamma^{\prime 2}-4 \delta^{\prime}<0$, since $\left|\delta^{\prime}\right|<1$, and if $\gamma^{\prime 2}-4 \delta^{\prime} \geq 0$ the inequality

$$
\frac{\left|\gamma^{\prime}\right|+\sqrt{\gamma^{\prime 2}-4 \delta^{\prime}}}{2}<1
$$

follows from the condition $\left|\gamma^{\prime}\right|<1+\delta^{\prime}$. Taking for $\alpha$ a zero of $P$ we obtain, by (23) and (26),

$$
M(\alpha)=\frac{M\left(P P^{\prime}\right)}{N \operatorname{cont} P}=\delta N\left(b, \beta^{\prime}\right) N \mathfrak{p}=\frac{\beta}{b} \cdot b p=p \beta .
$$

Now, assume (5) and let again $\delta=\beta / b$. In order to apply Lemma 3 we have to check (19).

Consider first the case

$$
\beta \notin\left\{\frac{1+\sqrt{4 e+1}}{2}: e \in \mathbb{N}\right\} .
$$

Then

$$
\beta-\left|\beta^{\prime}\right| \geq 2, \quad \beta \geq 1+\sqrt{2}
$$

and by $(24)$,

$$
R:=\frac{2 \sqrt{\delta}\left(1+\delta^{\prime}\right)}{N(1, \delta)}=2 \sqrt{\frac{\beta}{b}}\left(b+\beta^{\prime}\right) \geq 2 \sqrt{\beta}\left(\sqrt[4]{\beta\left|\beta^{\prime}\right|}+\operatorname{sgn} \beta^{\prime} \sqrt[4]{\left|\beta^{\prime}\right|^{3} / \beta}\right) .
$$

If $\beta^{\prime}>0$ we clearly have $R>1$, while if $\beta^{\prime}<0$ we have, by (26),

$$
R=2 \sqrt[4]{\beta\left|\beta^{\prime}\right|}\left(\sqrt{\beta}-\sqrt{\left|\beta^{\prime}\right|}\right) \geq 4 \sqrt[4]{\beta\left|\beta^{\prime}\right|} /\left(\sqrt{\beta}+\sqrt{\left|\beta^{\prime}\right|}\right) .
$$

If $\sqrt{\left|\beta^{\prime}\right|} \leq \frac{1}{2} \sqrt{\beta}$, it follows that

$$
R \geq \sqrt[4]{\beta\left|\beta^{\prime}\right|} \sqrt{\beta}>1
$$

while if $\sqrt{\left|\beta^{\prime}\right|}>\frac{1}{2} \sqrt{\beta}$, it follows that

$$
R>\frac{4}{\sqrt{2}} \frac{\sqrt{\beta}}{2 \sqrt{\beta}}=\sqrt{2}>1
$$


thus (27) implies

$$
\min \left\{N(1, \delta), 2 \sqrt{\delta}\left(1+\delta^{\prime}\right)\right\}=N(1, \delta)
$$

and (19) follows from (5).

Consider now the case

$$
\beta=\frac{1+\sqrt{4 e+1}}{2} .
$$

By (23), $b^{2}+b>e>b^{2}-b, b \mid e$, which implies $e=b^{2}$. On the other hand, $4 e+1=f^{2} \Delta$ for some $f \in \mathbb{N}$. The inequality

$$
p>\sqrt{\Delta}=\frac{\sqrt{4 b^{2}+1}}{f}
$$

implies by a tedious computation

$$
p \geq \frac{2 b+1}{f}>\frac{\sqrt{\Delta}}{2 \sqrt{\frac{\beta}{b}}\left(b+\beta^{\prime}\right)}=\frac{N(1, \delta) \sqrt{\Delta}}{\min \left\{N(1, \delta), 2 \sqrt{\delta}\left(1+\delta^{\prime}\right)\right\}},
$$

hence (19) holds.

The assumptions of Lemma 3 being satisfied there exists $\gamma \in K$ satisfying (26) and arguing as before we obtain

$$
p \beta=M(\alpha),
$$

where $\alpha$ is a zero of $x^{2}+\gamma x+\delta$.

Proof of Corollary 1. For $\beta=(3+\sqrt{5}) / 2$ the condition (4) is satisfied. Now, (2) is fulfilled by $p=2$ only, and (3) is fulfilled by $p=5$ and by $p \equiv \pm 1$ $(\bmod 5)$ only.

Proof of Corollary 2. Take a totally positive unit $\varepsilon>1$ of $K$ and a prime $p>\varepsilon$ that splits in $K$. Then by Theorem 2, $p \varepsilon \in \mathcal{M}$. Assume that the basal irreducible polynomial $f$ of $p \varepsilon$ has all its zeros in $K$. Hence

$$
f(x)=a\left(x \pm \frac{p \varepsilon}{a}\right)\left(x \pm \frac{p \varepsilon^{\prime}}{a}\right), \quad p \varepsilon>a>p \varepsilon^{\prime}, a \in \mathbb{N}
$$

and the condition $p^{2} / a \in \mathbb{Z}$ together with $p>\varepsilon$ implies $a=p$. However, for $a=p, f$ is not primitive.

EXAmple 1 . For $K=\mathbb{Q}(\sqrt{2})$ we can take

$$
p \varepsilon=21+14 \sqrt{2}=M\left(7 x^{4}+2 x^{3}+41 x^{2}+22 x+7\right) .
$$

Proof of Corollary 3. There are only finitely many totally positive integers $\beta$ of $K$, which are Perron numbers, but do not satisfy (4). 
REMARK 2. By a more complicated argument one can show that for $\beta$ totally positive, (3) implies (1) unless

$$
\sqrt[4]{N \beta}+\frac{\sqrt{\Delta}}{\sqrt[4]{N \beta}} \geq 4 \sqrt{\beta} \text { and } p<1+\frac{1}{2 \sqrt{\beta}}\left(\sqrt[4]{N \beta}+\frac{\sqrt{\Delta}}{\sqrt[4]{N \beta}}\right)
$$

ExAmple 2. Theorem 2 does not allow us to decide whether $1+\sqrt{17}$ $\in \mathcal{M}$. This question is open, as is a more general question, whether (3) implies (1).

\section{References}

[1] R. L. Adler and B. Marcus, Topological entropy and equivalence of dynamical systems, Mem. Amer. Math. Soc. 20 (1971), no. 219.

[2] D. W. Boyd, Inverse problems for Mahler's measure, in: Diophantine Analysis, J. Loxton and A. van der Poorten (eds.), London Math. Soc. Lecture Notes 109, Cambridge Univ. Press, 1986, 147-158.

[3] - Perron units which are not Mahler measures, Ergodic Theory Dynam. Systems 6 (1986), 485-488.

[4] - Reciprocal algebraic integers whose Mahler measures are non-reciprocal, Canad. Math. Bull. 30 (1987), 3-8.

[5] J. D. Dixon and A. Dubickas, The values of Mahler measures, Mathematika, to appear.

[6] A. Dubickas, Mahler measures close to an integer, Canad. Math. Bull. 45 (2002), 196-203.

[7] —, On numbers which are Mahler measures, Monatsh. Math. 141 (2004), 119-126.

[8] -, Mahler measures generate the largest possible groups, Math. Res. Lett., to appear.

[9] E. Hecke, Lectures on the Theory of Algebraic Numbers, Springer, 1981.

Institute of Mathematics

Polish Academy of Sciences

P.O. Box 21

00-956 Warszawa, Poland

E-mail: schinzel@impan.gov.pl 